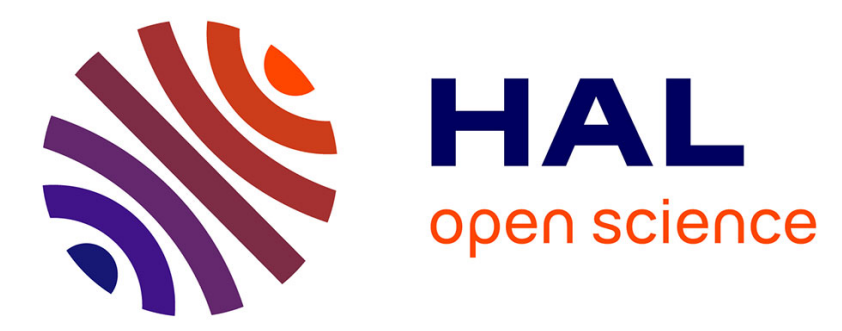

\title{
On State-dependent Discretization of Stable Homogeneous Systems
}

Denis Efimov, Andrey Polyakov, Alexander Yu Aleksandrov

\section{To cite this version:}

Denis Efimov, Andrey Polyakov, Alexander Yu Aleksandrov. On State-dependent Discretization of Stable Homogeneous Systems. CDC 2018 - 57th IEEE Conference on Decision and Control, Dec 2018, Miami Beach, FL, United States. 10.1109/CDC.2018.8618657 . hal-01888564

\section{HAL Id: hal-01888564 https://hal.inria.fr/hal-01888564}

Submitted on 5 Oct 2018

HAL is a multi-disciplinary open access archive for the deposit and dissemination of scientific research documents, whether they are published or not. The documents may come from teaching and research institutions in France or abroad, or from public or private research centers.
L'archive ouverte pluridisciplinaire HAL, est destinée au dépôt et à la diffusion de documents scientifiques de niveau recherche, publiés ou non, émanant des établissements d'enseignement et de recherche français ou étrangers, des laboratoires publics ou privés. 


\title{
On State-dependent Discretization of Stable Homogeneous Systems
}

\author{
D. Efimov, A. Polyakov, A. Yu. Aleksandrov
}

\begin{abstract}
Conditions for the existence and convergence to zero of numeric approximations with state-depend step of discretization to solutions of asymptotically stable homogeneous systems are obtained for the explicit and implicit Euler integration schemes. It is shown that for a sufficiently small discretization step the convergence of the approximating solutions to zero can be guaranteed globally in a finite or a fixed time, but in an infinite number of discretization iterations. It is proven that the absolute and relative errors of the respective discretizations are globally bounded functions. Efficiency of the proposed discretization algorithms is demonstrated by the simulation of the super-twisting system.
\end{abstract}

\section{INTRODUCTION}

Homogeneous dynamical systems found their place in the sliding mode control approaches [1], [2] and in the control or estimation solutions aiming on convergence in finite-time [3]-[9] or in fixedtime [10]-[12]. In addition, the popularity of homogeneous systems is based on the fact that they take an intermediate place between linear and nonlinear models [13] (the linear dynamical systems are also homogeneous). Therefore, from one side, the homogeneous systems admit some basic properties of linear dynamics (e.g. the scalability of trajectories), from another side, these systems are described by essentially nonlinear differential equations possessing such useful qualities as robustness to measurement noises, exogenous disturbances [14]-[16] and delays [17], [18], and increased rates of convergence to a goal invariant set (namely finite- or fixedtime convergence rates).

The industrial plants are frequently presented by continuoustime dynamical models, and a lot of control and estimation algorithms have been derived for such a class of systems, then the analysis or design is carried out in continuous time, while for a verification or implementation, the closed-loop system solutions have to be calculated in a computer or in a digital controller (for implementation of an observer, for instance). For these purposes different numerical discrete-time approximation methods and time discretization schemes are proposed for continuous-time differential equations [19], [20]. In this vein, the conventional Euler method is a first-order numerical routine for solving ordinary differential equations with a given initial value and a fixed time step, which represents the most basic explicit/implicit method of numerical integration and the simplest Runge-Kutta method. Since there are many control or estimation algorithms proposed recently, which are based on the theory of homogeneous systems (also possessing an increased rate of convergence with respect to linear systems), then implementation and derivation of solutions for such a class

D. Efimov and A. Polyakov are with Inria, Univ. Lille, CNRS, UMR 9189 - CRIStAL, F-59000 Lille, France.

A. Yu. Aleksandrov is with Department of Control of Complex Systems, University ITMO, 49 av. Kronverkskiy, 197101 Saint Petersburg, Russia.

This work was partially supported by ANR 15 CE23 0007 (Project Finite4SoS), the Government of Russian Federation (Grant 08-08) and the Ministry of Education and Science of Russian Federation (Project 14.Z50.31.0031). of systems become important being in the focus of many works, especially in the sliding mode control community. In particular, in the recent papers [21], [22] it has been shown that application of the explicit Euler method (with a fixed time step) for the global approximation of solutions of homogeneous systems with non-zero degree is problematic (see also [23]), and the implicit Euler scheme has a better perspective (see also [24]-[27]). In other papers, the conditions of convergence and stability of the explicit and implicit Euler methods have been studied for linear systems (the notion of A-stability) [20], [28], [29], or for particular classes of nonlinear systems [30], [31].

This paper continues the line of research initiated in [21] and it is devoted to application of explicit and implicit Euler discretization schemes for approximation of solutions of homogeneous stable dynamical systems. In the present work, the case with a state-dependent time step is considered and different conditions for the existence and convergence to zero of solutions for the explicit and implicit Euler integration schemes are obtained for homogeneous systems (by repeating the same analysis steps as in [21]). It is shown that introduction of state-dependent re-scaling of the time discretization step allows the finite- or fixed-time rates of convergence to be recovered for the discrete-time approximations of solutions, but for an infinite number of steps (in a finite number of steps the convergence to a vicinity of the origin can be obtained). Absolute and relative errors (closeness of the approximations to real solutions) for the explicit and implicit Euler integration schemes are investigated using the homogeneity theory, and it is shown that the relative errors are globally bounded and by decreasing the initial discretization step it is possible to make them arbitrary small.

The outline of this note is as follows. The problem statement and some preliminary results are introduced in section II. Some basic properties and relations between solution approximations are studied in Section III. The convergence conditions are established in Section IV. Properties of relative and absolute errors of approximation of solutions of homogeneous systems by the explicit and implicit Euler methods are investigated in Section V. A simple illustrating example (the super-twisting system) is considered in Section VI.

\section{Notation}

- $\mathbb{R}_{+}=\{x \in \mathbb{R}: x \geq 0\}$, where $\mathbb{R}$ is the set of real number.

- $|\cdot|$ denotes the absolute value in $\mathbb{R}$, for a vector $x \in \mathbb{R}^{n}$ the symbol $\|x\|$ denotes the Euclidean norm, and the corresponding induced matrix norm $\|A\|$ for a matrix $A \in \mathbb{R}^{n \times n}$.

- A continuous function $\alpha: \mathbb{R}_{+} \rightarrow \mathbb{R}_{+}$belongs to the class $\mathcal{K}$ if $\alpha(0)=0$ and the function is strictly increasing. The function $\alpha: \mathbb{R}_{+} \rightarrow \mathbb{R}_{+}$belongs to the class $\mathcal{K}_{\infty}$ if $\alpha \in \mathcal{K}$ and it is increasing to infinity.

- The identity matrix of dimension $n \times n$ is denoted as $I_{n}$, and $\operatorname{diag}\left\{r_{i}\right\}_{i=1}^{n}$ is a diagonal matrix with the elements on the main diagonal equal $r_{i}$.

- A sequence of integers $1,2, \ldots, n$ is denoted by $\overline{1, n}$. 


\section{PRELIMINARIES}

In this work the following nonlinear system is considered:

$$
\dot{x}(t)=f(x(t)), t \geq 0,
$$

where $x(t) \in \mathbb{R}^{n}$ is the state, $f: \mathbb{R}^{n} \rightarrow \mathbb{R}^{n}$ ensures forward existence and uniqueness of the system solutions at least locally, $f(0)=0$. For an initial condition $x_{0} \in \mathbb{R}^{n}$ define the corresponding solution by $X\left(t, x_{0}\right)$ for any $t \geq 0$ for which the solution exists. If $f$ is discontinuous, then the solutions are understood in the Filippov's sense [32].

Following [11], [33], [34], let $\Omega \subset \mathbb{R}^{n}$ be an open set, $0 \in \Omega$.

Definition 1. At the steady state $x=0$ the system (1) is said to be

(a) stable if for any $x_{0} \in \Omega$ the solution $X\left(t, x_{0}\right)$ is defined for all $t \geq 0$, and for any $\epsilon>0$ there is $\delta>0$ such that for any $x_{0} \in \Omega$, if $\left\|x_{0}\right\| \leq \delta$ then $\left\|X\left(t, x_{0}\right)\right\| \leq \epsilon$ for all $t \geq 0$;

(b) asymptotically stable if it is stable and for any $\kappa>0$ and $\epsilon>0$ there exists $T(\kappa, \epsilon) \geq 0$ such that for any $x_{0} \in \Omega$, if $\left\|x_{0}\right\| \leq \kappa$ then $\left\|X\left(t, x_{0}\right)\right\| \leq \epsilon$ for all $t \geq T(\kappa, \epsilon)$;

(c) finite-time stable if it is stable and finite-time converging from $\Omega$, i.e. for any $x_{0} \in \Omega$ there exists $0 \leq T<+\infty$ such that $X\left(t, x_{0}\right)=0$ for all $t \geq T$. The function $T_{0}\left(x_{0}\right)=\inf \{T \geq 0$ : $\left.X\left(t, x_{0}\right)=0 \forall t \geq T\right\}$ is called the settling time of the system (1);

$(d)$ fixed-time stable if it is finite-time stable and $\sup _{x_{0} \in \Omega} T_{0}\left(x_{0}\right)<+\infty$.

The set $\Omega$ is called a domain of stability/attraction.

If $\Omega=\mathbb{R}^{n}$, then the corresponding properties are called global stability/asymptotic stability/finite-time/fixed-time stability of (1) at $x=0$.

Similarly, the stability notions can be defined with respect to a compact invariant set, by replacing the distance to the origin in Definition 1 with the distance to a set.

\section{A. Weighted homogeneity}

Following [1], [13], [35], for strictly positive numbers $r_{i}, i=\overline{1, n}$ called weights and $\lambda>0$, define:

- the vector of weights $\mathbf{r}=\left(r_{1}, \ldots, r_{n}\right)^{\top}, r_{\max }=$ $\max _{1 \leq j \leq n} r_{j}$ and $r_{\min }=\min _{1 \leq j \leq n} r_{j}$;

- the dilation matrix function $\Lambda_{r}(\bar{\lambda})=\operatorname{diag}\left\{\lambda^{r_{i}}\right\}_{i=1}^{n}$, note that $\forall x \in \mathbb{R}^{n}$ and $\forall \lambda>0$ we have $\Lambda_{r}(\lambda) x=$ $\left(\lambda^{r_{1}} x_{1}, \ldots, \lambda^{r_{n}} x_{n}\right)^{\top}$;

- the $\mathbf{r}$-homogeneous norm $\|x\|_{r}=\left(\sum_{i=1}^{n}\left|x_{i}\right|^{\frac{\rho}{r_{i}}}\right)^{\frac{1}{\rho}}$ for any $x \in \mathbb{R}^{n}$ and $\rho \geq r_{\max }$, it is not a norm in the standard sense, since the triangle inequality is not satisfied for $\|\cdot\|_{r}$, however there exist $\underline{\sigma}, \bar{\sigma} \in \mathcal{K}_{\infty}$ such that

$$
\underline{\sigma}\left(\|x\|_{r}\right) \leq\|x\| \leq \bar{\sigma}\left(\|x\|_{r}\right) \quad \forall x \in \mathbb{R}^{n} ;
$$

- the sphere and the ball in the homogeneous norm $S_{r}(\rho)=$ $\left\{x \in \mathbb{R}^{n}:\|x\|_{r}=\rho\right\}$ and $B_{r}(\rho)=\left\{x \in \mathbb{R}^{n}:\|x\|_{r} \leq \rho\right\}$ for $\rho \geq 0$.

Definition 2. A function $g: \mathbb{R}^{n} \rightarrow \mathbb{R}$ is $\mathbf{r}$-homogeneous with degree $\mu \in \mathbb{R}$ if $\forall x \in \mathbb{R}^{n}$ and $\forall \lambda>0$ we have:

$$
\lambda^{-\mu} g\left(\Lambda_{r}(\lambda) x\right)=g(x) .
$$

A vector field $f: \mathbb{R}^{n} \rightarrow \mathbb{R}^{n}$ is $\mathbf{r}$-homogeneous with degree $\nu \in \mathbb{R}$, with $\nu \geq-r_{\min }$ if $\forall x \in \mathbb{R}^{n}$ and $\forall \lambda>0$ we have:

$$
\lambda^{-\nu} \Lambda_{r}^{-1}(\lambda) f\left(\Lambda_{r}(\lambda) x\right)=f(x),
$$

which is equivalent for $i$-th component of $f$ being a $\mathbf{r}$-homogeneous function of degree $r_{i}+\nu$.

System (1) is $\mathbf{r}$-homogeneous of degree $\nu$ if the vector field $f$ is $\mathbf{r}$-homogeneous of the degree $\nu$.

The homogeneous norm $\|\cdot\|_{r}$ is an example of $\mathbf{r}$-homogeneous function with degree 1 .

Theorem 1. [35], [36] For the system (1) with $\mathbf{r}$-homogeneous and continuous function $f$ the following properties are equivalent:

- the system (1) is asymptotically stable;

- there exists a smooth $\mathbf{r}$-homogeneous Lyapunov function $V$ : $\mathbb{R}^{n} \rightarrow \mathbb{R}_{+}$such that

$$
\begin{gathered}
\alpha_{1}(\|x\|) \leq V(x) \leq \alpha_{2}(\|x\|), \\
L_{f} V(x)=\frac{\partial V}{\partial x}(x) f(x) \leq-\alpha(\|x\|), \\
\lambda^{-\mu} V\left(\Lambda_{r}(\lambda) x\right)=V(x), \mu>r_{\max },
\end{gathered}
$$

$\forall x \in \mathbb{R}^{n}$ and $\forall \lambda>0$, for some $\alpha_{1}, \alpha_{2} \in \mathcal{K}_{\infty}$ and $\alpha \in \mathcal{K}$.

The requirement on continuity of the function $f$ has been relaxed in [37] (the function $V$ can still be selected smooth).

\section{EULER SCHEMES}

Let us consider a class of systems in (1) with

$$
\sup _{\xi \in S_{r}(1)}\|f(\xi)\|<+\infty
$$

and admitting the following hypothesis:

Assumption 1. Let (1) be $\mathbf{r}$-homogeneous with a degree $\nu$ and asymptotically stable.

In this work, in order to obtain a discretization of the solutions of (1), we will propose modifications of implicit and explicit Euler methods [20] with state-dependent scaling of the time discretization step. To this end, select a basic discretization step $h>0$, define a sequence of time instants $t_{i}$ for $i=0,1, \ldots$ such that $t_{0}=0$ and $t_{i+1}-t_{i}>0$, and denote by $x_{i}$ an approximation of the solution $X\left(t_{i}, x_{0}\right)$ at the corresponding time instant (i.e. $x_{i} \simeq X\left(t_{i}, x_{0}\right)$ and $x_{0}=X\left(0, x_{0}\right)$ ), then the approximation $x_{i+1}$ calculated in accordance with the explicit Euler method is given by:

$$
\begin{aligned}
x_{i+1} & =x_{i}+\frac{h}{\left\|x_{i}\right\|_{r}^{\nu}} f\left(x_{i}\right), \\
t_{i+1} & =t_{i}+\frac{h}{\left\|x_{i}\right\|_{r}^{\nu}}
\end{aligned}
$$

for $i=0,1, \ldots$, while the approximation calculated by the implicit Euler method comes from:

$$
\begin{aligned}
x_{i+1} & =x_{i}+\frac{h}{\left\|x_{i+1}\right\|_{r}^{\nu}} f\left(x_{i+1}\right), \\
t_{i+1} & =t_{i}+\frac{h}{\left\|x_{i+1}\right\|_{r}^{\nu}}
\end{aligned}
$$

for $i=0,1, \ldots$

It is a well-known fact that for $\nu=0$ with $h \rightarrow 0$ both methods approach the real solution [20], i.e. $x_{i} \rightarrow X\left(t_{i}, x_{0}\right)$ in (2) and (3) with $h \rightarrow 0$ over any compact time interval. In [21] for the case of the conventional explicit and implicit Euler methods with a constant discretization step $h$ and $\nu \neq 0$ it has been shown that outside of a vicinity of the origin for $\nu<0$ or a vicinity of infinity for $\nu>0$ the same results can be obtained. 
In the sequel, the problem of convergence to zero of the approximations $\left\{x_{i}\right\}_{i=0}^{\infty}$ derived in (2) and (3) is studied for systems in (1) satisfying Assumption 1 with $\nu \neq 0$ (the case $\nu=0$, or without scaling of the discretization step, has been analyzed in [21]). For this goal we need to establish some auxiliary properties of approximations $\left\{x_{i}\right\}_{i=0}^{\infty}$ obtained in (2) and (3).

\section{A. Relations between approximations obtained for different initial conditions}

One of the main features of (2) and (3) is as follows:

Proposition 1. Let the system (1) be $\mathbf{r}$-homogeneous with a degree $\nu$. If $\left\{x_{i}\right\}_{i=0}^{\infty}$ is a sequence generated by (2) or (3) for the time instants $\left\{t_{i}\right\}_{i=0}^{\infty}$ with the step $h$ and the initial state $x_{0}$, then for any $\lambda>0, y_{i}=\Lambda_{r}(\lambda) x_{i}$ is a sequence obtained by (2) or (3), respectively, for the instants $\lambda^{-\nu}\left\{t_{i}\right\}_{i=0}^{\infty}$ with the step $h$ and the initial state $y_{0}=\Lambda_{r}(\lambda) x_{0}$.

All proofs are excluded due to space limitations.

Note that $y_{i}$ is an approximation of $X\left(\lambda^{-\nu} t_{i}, y_{0}\right)$ for scaled instants of time. The following corollaries can be established.

Corollary 1. Let the system (1) be $\mathbf{r}$-homogeneous with a degree $\nu$. Let for all $x_{0} \in S_{r}(1)$ there exist sequences $\left\{x_{i}\right\}_{i=0}^{\infty}$ obtained by (2) or (3) with the step $h>0$ and the initial state $x_{0}$ possessing one of the following properties:

$$
\begin{gathered}
\sup _{i \geq 0}\left\|x_{i}\right\|<+\infty \\
\lim _{i \rightarrow+\infty} x_{i}=0 .
\end{gathered}
$$

Then for any $y_{0} \in \mathbb{R}^{n}$ there exist sequences $\left\{y_{i}\right\}_{i=0}^{\infty}$ generated by (2) or (3) with the step $h$ and the initial state $y_{0}$ possessing the same property.

\section{B. Norm deviations}

Another important property of the proposed approximation algorithms (2) and (3) consists in their finite increment:

Lemma 1. Let the system (1) be $\mathbf{r}$-homogeneous with a degree $\nu$. Then there are $h_{0}>0$ and $0<\underline{c}<\bar{c}<+\infty$ such that for any $h \in\left(0, h_{0}\right]$ :

$$
\underline{c}\left\|x_{i}\right\|_{r} \leq\left\|x_{i+1}\right\|_{r} \leq \bar{c}\left\|x_{i}\right\|_{r}
$$

for all $x_{i} \in \mathbb{R}^{n}$ in (2) or (3) (provided that a solution $x_{i+1}$ exists).

Then the following result can be derived:

Corollary 2. Let the system (1) be $\mathbf{r}$-homogeneous with a degree $\nu$. Select $h_{0}>0$, then there exists $0<c<+\infty$ such that for any $h \in\left(0, h_{0}\right]$ and for all $x_{i} \in \mathbb{R}^{n},\left\|x_{i+1}\right\|_{r} \leq c\left\|x_{i}\right\|_{r}$ in (2) or $c\left\|x_{i}\right\|_{r} \leq\left\|x_{i+1}\right\|_{r}$ in (3) (provided that a solution $x_{i+1}$ exists).

\section{CONVERGEnce OF SEQUENCES $\left\{x_{i}\right\}_{i=0}^{\infty}$ GENERATED By EULER METHODS}

In this section we will study the stability and convergence features of $\left\{x_{i}\right\}_{i=0}^{\infty}$ only. The quality of the corresponding approximations (the closeness of $\left\{x_{i}\right\}_{i=0}^{\infty}$ to the continuous-time solutions $\left.X\left(t_{i}, x_{0}\right)\right)$ will be considered in the next section.
According to Theorem 1, under Assumption 1 for the system (1) there is a twice continuously differentiable and $\mathbf{r}$-homogeneous Lyapunov function $V: \mathbb{R}^{n} \rightarrow \mathbb{R}_{+}$of the degree $\mu>-\nu$ such that

$$
\begin{gathered}
a=-\sup _{\xi \in S_{r}(1)} L_{f} V(\xi)>0, \\
0<b=\sup _{\xi \in B_{r}(1)}\left\|\frac{\partial V(\xi)}{\partial \xi}\right\|<+\infty, \\
c_{1}=\inf _{\xi \in S_{r}(1)} V(\xi), c_{2}=\sup _{\xi \in S_{r}(1)} V(\xi), \\
c_{1}\|x\|_{r}^{\mu} \leq V(x) \leq c_{2}\|x\|_{r}^{\mu} \quad \forall x \in \mathbb{R}^{n} .
\end{gathered}
$$

\section{A. Convergence of the explicit Euler scheme (2)}

The main result of this subsection is as follows:

Theorem 2. Let Assumption 1 be satisfied, then there exists $h_{0}>$ 0 such that for any discretization step $h \in\left(0, h_{0}\right]$ the sequences $\left\{x_{i}\right\}_{i=0}^{\infty}$ obtained by (2) for any initial state $x_{0} \in \mathbb{R}^{n}$ and the step $h$ possess the following properties:

(a) $\sup _{i=0,1, \ldots}\left\|x_{i}\right\|_{r}<\gamma\left\|x_{0}\right\|_{r}$ for some $\gamma \in(0,+\infty)$;

(b) $\lim \sup _{i \rightarrow+\infty}\left\|x_{i}\right\|_{r}=0$;

(c) for $\nu=0$ the sequence $\left\{x_{i}\right\}_{i=0}^{\infty}$ has an exponential convergence rate, for $\nu<0$ for any $x_{0} \in \mathbb{R}^{n}$ the time of convergence to the origin $t_{+\infty}^{x_{0}}=\lim _{t \rightarrow+\infty} t_{i}$ is finite, and for $\nu>0$ the time of convergence from any initial conditions $x_{0} \in \mathbb{R}^{n}$ to $B_{r}(1)$ is also finite independently of $x_{0}$.

Note that an estimate on $h_{0}$ is provided in the proof of Theorem 2, and repeating the same arguments it can be proven the existence of $h_{1} \in\left(h_{0},+\infty\right)$ such that for all $h \geq h_{1}$ the discretization scheme is diverging. The property obtained for $\nu>0$ is related with the fixed-time rate of convergence (to the unit ball).

\section{B. Existence of approximations in (3)}

Existence of some $x_{i+1} \in \mathbb{R}^{n}$ for any $x_{i} \in \mathbb{R}^{n}$ in the explicit case (2) is straightforward, but it is not the case of (3). According to the result of Corollary 1 , it is enough to find the conditions of existence of $x_{i+1}$ for all $x_{i} \in S_{r}(1)$ in (3). Note that in a general case, it is difficult to provide some simple conditions for existence and uniqueness of $x_{i+1}$ in the equation (3) for any $x_{0} \in S_{r}(1)$, but homogeneity may further simplify the solution under additional mild restrictions on $f$.

Proposition 2. Let $f$ be $\mathbf{r}$-homogeneous of degree $\nu$ and continuous on $S_{r}(1)$. Then there is $h_{0}>0$ such that for all $h \in\left(0, h_{0}\right)$ the equation (3) for any $x_{i} \in \mathbb{R}^{n}$ has a solution $x_{i+1} \in \mathbb{R}^{n}$.

Thus, for the proposed implicit Euler method with statedependent discretization step (3), the global existence of solutions may be provided by selecting $h$ sufficiently small provided that $f$ is homogeneous and continuous.

\section{Convergence of the implicit Euler scheme (3)}

Similar to (2) results can be obtained for (3) and even with some improvement under additional restrictions. Note that in this subsection we assume that the sequences $\left\{x_{i}\right\}_{i=0}^{\infty}$ exist in (3) for all $x_{0} \in \mathbb{R}^{n}$, e.g. the conditions of Proposition 2 are verified.

Theorem 3. Let Assumption 1 be satisfied, then there exists $h_{0}>$ 0 such that for any discretization step $h \in\left(0, h_{0}\right]$ the sequences $\left\{x_{i}\right\}_{i=0}^{\infty}$ obtained by (3) for any initial state $x_{0} \in \mathbb{R}^{n}$ and the step $h$ possess the following properties: 
(a) $\sup _{i=0,1, \ldots}\left\|x_{i}\right\|_{r}<\gamma\left\|x_{0}\right\|_{r}$ for some $\gamma \in(0,+\infty)$;

(b) $\lim \sup _{i \rightarrow+\infty}\left\|x_{i}\right\|_{r}=0$;

(c) for $\nu=0$ the sequence $\left\{x_{i}\right\}_{i=0}^{\infty}$ has an exponential convergence rate, for $\nu<0$ for any $x_{0} \in \mathbb{R}^{n}$ the time of convergence to the origin $t_{+\infty}^{x_{0}}=\lim _{t \rightarrow+\infty} t_{i}$ is finite, and for $\nu>0$ the time of convergence from any initial conditions $x_{0} \in \mathbb{R}^{n}$ to $B_{r}(1)$ is also finite independently of $x_{0}$.

If the matrix $\frac{\partial^{2} V(\xi)}{\partial \xi^{2}} \geq 0$ for all $\xi \in \mathbb{R}^{n}$, where $V: \mathbb{R}^{n} \rightarrow \mathbb{R}_{+}$ is a $\mathbf{r}$-homogeneous Lyapunov function satisfying (6), then $h_{0}>0$ can be selected arbitrary.

The requirement on nonnegative definiteness of the second derivative of $V$ is related with the condition of convexity level set of $V$ imposed in [21].

\section{Absolute and Relative ERrors of Discretized HOMOGENEOUS SYSTEMS}

Conventional characteristics of the discretization accuracy are studied in this section for the proposed algorithms (2) or (3) and for homogeneous systems. To this end denote $\Xi_{h}\left(x_{0}\right)=$ $X\left(h\left\|x_{0}\right\|_{r}^{-\nu}, x_{0}\right)$ as the value of the solution of (1) with the initial condition $x_{0} \in \mathbb{R}^{n}$ evaluated in (2) or (3) after one iteration with the discretization step $h>0$ (at $t_{1}=h\left\|x_{0}\right\|_{r}^{-\nu}$ with $t_{0}=0$ ). Denote by $\hat{\Xi}_{h}\left(x_{0}\right)$ the estimated value derived by (2) or (3) for the same $x_{0}$ and $h>0$ (note that $\hat{\Xi}_{h}\left(x_{0}\right)=x_{0}+\frac{h}{\left\|x_{0}\right\|_{r}^{\nu}} f\left(x_{0}\right)$ in the case of (2)), then [38]:

- the absolute error is the magnitude of the difference between the exact value and its approximation:

$$
\begin{gathered}
\Delta^{h}(x)=\left\|\Xi_{h}(x)-\hat{\Xi}_{h}(x)\right\|, \\
\Delta_{r}^{h}(x)=\left\|\Xi_{h}(x)-\hat{\Xi}_{h}(x)\right\|_{r} ;
\end{gathered}
$$

- the relative error expresses how large the absolute error is compared with the exact value:

$$
\delta^{h}(x)=\frac{\Delta^{h}(x)}{\left\|\Xi_{h}(x)\right\|}, \delta_{r}^{h}(x)=\frac{\Delta_{r}^{h}(x)}{\left\|\Xi^{h}(x)\right\|_{r}} .
$$

The errors are given for two different norms, the conventional one $\|\cdot\|$ and the homogeneous norm $\|\cdot\|_{r}$, the former one is used habitually for evaluation of a discretization method precision, while the latter norm suits better for analysis of homogeneous systems. Equivalence of these errors for different norms have been shown in [21], that is why in this note we will study only $\Delta_{r}^{h}\left(x_{i}\right)$ and $\delta_{r}^{h}\left(x_{i}\right)$.

These error functions admit the following useful properties:

Theorem 4. Let the system (1) be $\mathbf{r}$-homogeneous of degree $\nu$ and $\hat{\Xi}_{h}(x)$ be calculated by the explicit (2) or implicit (3) Euler scheme for $x \in \mathbb{R}^{n}$ and $h>0$. Then the functions $\Delta_{r}^{h}(x)$ and $\delta_{r}^{h}(x)$ are $\mathbf{r}$-homogeneous of degree 1 and 0 , respectively.

Any homogeneous function of degree 0 is globally bounded (it may be discontinuous) if its maximal amplitude is finite being evaluated on $S_{r}(1)$. Therefore, if for any initial conditions $x_{0} \in S_{r}(1)$ the error $\delta_{r}^{h}(x)$ stay sufficiently small for a reasonable selection of $h$ (i.e. the one step error of usual Euler discretization approaches is small on the sphere), then the explicit (2) and the implicit (3) Euler schemes provide a uniformly bounded relative error $\delta_{r}$ globally. Boundedness of $\delta_{r}$ implies that the difference between $\Xi_{h}(x)$ and $\hat{\Xi}_{h}(x)$ stays of the order $\Xi_{h}(x)$ (roughly speaking proportional to $x)$.

This is an interesting and important observation motivating the use of (2) and (3) in the applications. Let us consider one of them.

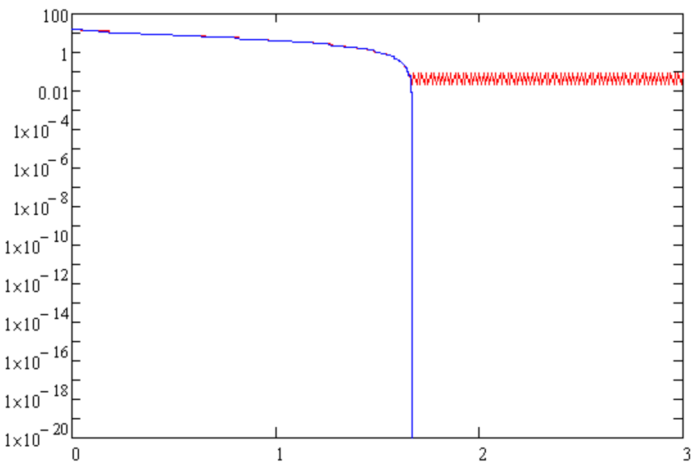

Figure 1. The results of simulation for $h=0.01$

Remark 1. Note that the time step in (2) and (3) is state- and degreedependent. In particular, if $\left\|x_{i}\right\|_{r} \gg 1$ (it is sufficiently big) and $\nu<$ 0 , then $t_{i+1}-t_{i}=\left\|x_{i}\right\|_{r}^{-\nu} h \gg h$ in (2) and the time step can be too large, which is also related with the obtained accuracy estimates in Theorem 4. It is worth stressing that anyway the convergence of these algorithms is not influenced, and this observation deals only with the precision. Therefore, for big amplitudes of $x_{i}$ in the case of $\nu<0$ it is desirable to use the conventional Euler methods (without a scaling of the time discretization step), which may provide a better accuracy of approximation of the solutions [21], [22].

\section{EXAMPLE}

Consider the super-twisting benchmark system:

$$
\begin{aligned}
& \dot{x}_{1}=-k_{1}\left|x_{1}\right|^{0.5} \operatorname{sign}\left(x_{1}\right)+x_{2}, \\
& \dot{x}_{2}=-k_{2} \operatorname{sign}\left(x_{1}\right),
\end{aligned}
$$

where $x=\left[\begin{array}{ll}x_{1} & x_{2}\end{array}\right]^{\top} \in \mathbb{R}^{2}$ is the state vector, $k_{1}>0$ and $k_{2}>0$ are the system parameters, $\nu=-0.5$ is the homogeneity degree for $\mathbf{r}=\left[\begin{array}{ll}1 & 0.5\end{array}\right]^{\top}$. Since it is a discontinuous system, then we are going to compare for this model the explicit method (2) and the same approach without state-dependent scaling:

$$
x_{i+1}=x_{i}+h f\left(x_{i}\right), t_{i}=i h
$$

for $i=0,1, \ldots$, which was studied in many works previously and it is recommended for the use in the sliding mode control community [1], [21]-[23] (a more sophisticated solution is given in [39]).

For simulations $k_{1}=10$ and $k_{2}=6$ have been selected, and the run of the algorithms (2) and (7) have been performed for the same initial condition $x_{0}=\left[\begin{array}{ll}10 & 10\end{array}\right]^{\top}$ with two values of discretization step $h: 10^{-2}$ and $10^{-3}$ (see the figures 1 and 2, respectively, where $|x(t)|$ is plotted in a logarithmic scale, the results of (2) correspond to the blue lines, the results of (7) are presented by the red ones). As we can conclude from these simulations, the method (2) outperforms drastically (7) (the former is finite-time converging, while the latter one cannot converge to the origin) having a similar computational complexity (comparing with (7), in (2) it is necessary to additionally compute the value of the norm $\left\|x_{i}\right\|_{r}^{\nu}$, which only needs the power and the addition operations), that confirms the theoretical finding of this work.

\section{CONCLUSIONS}

In this work a set of results has been obtained devoted to application of the explicit and implicit Euler methods (with state-dependent scaling of the discretization step) for discrete-time approximation of 


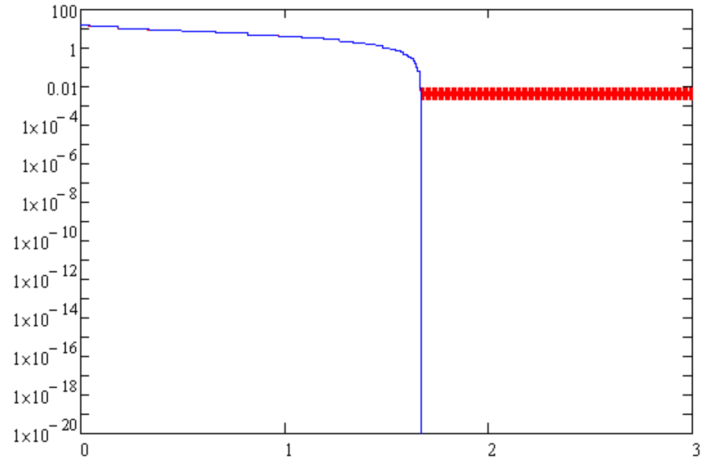

Figure 2. The results of simulation for $h=0.001$

homogeneous systems. The main contributions can be summarized as follows:

- The proposed algorithms (2) and (3) possess certain homogeneity, and if the convergence is ensured on the sphere, then by scaling it can be extended globally (Proposition 1).

- The obtained approximations can be made globally converging for asymptotically stable homogeneous systems provided that the discretization step is selected sufficiently small, in addition they preserve finite-time or fixed-time rates of convergence in infinite number of iterations (theorems 2 and 3).

- For the implicit Euler scheme it has been proved, under an additional mild condition, that solutions always exist for a sufficiently small discretization steps (Proposition 2). In addition, the approximations are converging to zero for any initial conditions and discretization steps if the Hessian of the Lyapunov function of the system is nonnegative definite (Theorem 3).

- For any value of the discretization step, the implicit and explicit Euler methods provide a good approximation of the system solutions (Theorem 4).

- For $\nu<0$ the explicit Euler method with a constant step (7) can be used outside of a vicinity of the origin and next switching to the explicit (2) or the implicit (3) Euler methods is reasonable, in order to demonstrate convergence to the origin (initial application of the explicit method is motivated by its lower computational complexity and a good accuracy).

Future directions of research will include analysis of applicability of Euler methods for locally homogeneous systems, as well as investigation of its use in applications and for implementation of controllers and observers.

\section{REFERENCES}

[1] A. Levant, "Homogeneity approach to high-order sliding mode design," Automatica, vol. 41, no. 5, pp. 823-830, 2005.

[2] E. Bernuau, D. Efimov, W. Perruquetti, and A. Polyakov, "On homogeneity and its application in sliding mode," Int. J. Franklin Institute, vol. 351, no. 4, pp. 1866-1901, 2014.

[3] L. Grüne, "Homogeneous state feedback stabilization of homogeneous systems," SIAM J. Control Optimization, vol. 38, no. 4, pp. 1288-1314, 2000.

[4] Y. Hong, " $\mathrm{H}_{\infty}$ control, stabilization, and input-output stability of nonlinear systems with homogeneous properties," Automatica, vol. 37, no. 7, pp. 819-829, 2001.

[5] S. P. Bhat and D. S. Bernstein, "Geometric homogeneity with applications to finite-time stability," Mathematics of Control, Signals and Systems, vol. 17, pp. 101-127, 2005.
[6] E. Moulay and W. Perruquetti, "Finite time stability of differential inclusions," IMA J. Math. Control Inform, vol. 22, no. 4, pp. 465-475, 2005.

[7] J. Li and C. Qian, "Global finite-time stabilization by dynamic output feedback for a class of continuous nonlinear systems," IEEE Transactions on Automatic Control, vol. 51, no. 5, pp. 879-884, 2006.

[8] Y. Shen and X. Xia, "Semi-global finite-time observers for nonlinear systems," Automatica, vol. 44, no. 12, pp. 3152-3156, 2008.

[9] H. Yiguang, J. Zhong-Ping, and F. Gang, "Finite-time input-to-state stability and applications to finite-time control design," SIAM J. Control Optim., vol. 48, no. 7, pp. 4395-4418, 2010.

[10] E. Cruz-Zavala, J. Moreno, and L. Fridman, "Uniform robust exact differentiator," IEEE Transactions on Automatic Control, vol. 56, no. 11, pp. 2727-2733, 2011.

[11] A. Polyakov, "Nonlinear feedback design for fixed-time stabilization of linear control systems," IEEE Transactions on Automatic Control, vol. 57, no. 8, pp. 2106-2110, 2012.

[12] A. Polyakov, D. Efimov, and W. Perruquetti, "Finite-time and fixedtime stabilization: Implicit lyapunov function approach," Automatica, vol. 51, pp. 332-340, 2015.

[13] A. Bacciotti and L. Rosier, Lyapunov Functions and Stability in Control Theory, 2nd ed. Springer, Berlin, 2005.

[14] A. Aleksandrov, "On the asymptotical stability of solutions of nonstationary differential equation systems with homogeneous right hand sides," Dokl. Akad. Nauk Rossii, vol. 349, no. 3, pp. 295-296, 1996, in Russian.

[15] J. Peuteman and D. Aeyels, "Averaging results and the study of uniform asymptotic stability of homogeneous differential equations that are not fast time-varying," SIAM J. Control Optim, vol. 37, no. 4, pp. 9971010, 1999.

[16] E. Bernuau, A. Polyakov, D. Efimov, and W. Perruquetti, "Verification of ISS, iISS and IOSS properties applying weighted homogeneity," Systems \& Control Letters, vol. 62, pp. 1159-1167, 2013.

[17] D. Efimov, A. Polyakov, W. Perruquetti, and J.-P. Richard, "Weighted homogeneity for time-delay systems: Finite-time and independent of delay stability," IEEE Transactions on Automatic Control, vol. 61, no. 1, pp. 210-215, 2016.

[18] K. Zimenko, D. Efimov, A. Polyakov, and W. Perruquetti, "A note on delay robustness for homogeneous systems with negative degree," Automatica, vol. 79, no. 5, pp. 178-184, 2017.

[19] M. Allen and E. Isaacson, Numerical Analysis for Applied Science, ser. A Wiley-Interscience publication. Wiley, 1998.

[20] J. C. Butcher, Numerical Methods for Ordinary Differential Equations, 2nd ed. New York: John Wiley \& Sons, 2008.

[21] D. Efimov, A. Polyakov, A. Levant, and W. Perruquetti, "Realization and discretization of asymptotically stable homogeneous systems," IEEE Transactions on Automatic Control, vol. 62, no. 11, pp. 59625969, 2017.

[22] A. Levant, D. Efimov, A. Polyakov, and W. Perruquetti, "Stability and robustness of homogeneous differential inclusions," in Proc. IEEE CDC, 2016

[23] A. Levant, "On fixed and finite time stability in sliding mode control," in IEEE 52nd Annual Conference on Decision and Control (CDC), 2013, pp. 4260-4265.

[24] V. Acary and B. Brogliato, "Implicit euler numerical simulations of sliding mode systems," System and Control Letters, vol. 59, no. 5, pp. 284-293, 2010.

[25] B. Brogliato and A. Polyakov, "Globally stable implicit euler timediscretization of a nonlinear single-input sliding-mode control system," in IEEE CDC, 2015, pp. 5426-5431.

[26] O. Huber, V. Acary, and B. Brogliato, "Lyapunov stability and performance analysis of the implicit discrete sliding mode control," IEEE Transactions on Automatic Control, vol. 61, no. 10, pp. 3016-3030, 2016.

[27] F. A. Miranda-Villatoro, B. Brogliato, and F. Castanos, "Multivalued robust tracking control of lagrange systems: Continuous and discretetime algorithms," IEEE Transactions on Automatic Control, vol. 62, no. 9, pp. 4436-4450, 2017.

[28] G. G. Dahlquist, "A special stability problem for linear multistep methods," BIT Numerical Mathematics, vol. 3, no. 1, pp. 27-43, 1963.

[29] A. Aleksandrov and A. Zhabko, "Preservation of stability under discretization of systems of ordinary differential equations," Siberian Math. J., vol. 51, no. 3, pp. 383-395, 2010. 
[30] C. Gonzalez, A. Ostermann, C. Palencia, and M. Thalhammer, "Backward euler discretization of fully nonlinear parabolic problems," Mathematics of Computation, vol. 71, no. 237, pp. 125-145, 2002. [Online]. Available: http://www.jstor.org/stable/2698863

[31] B. Merlet and M. Pierre, "Convergence to equilibrium for the backward euler scheme and applications," Communications on Pure and Applied Analysis, vol. 9, no. 3, pp. 685-702, 2010.

[32] A. Filippov, Differential Equations with Discontinuous Righthand Sides. Kluwer Academic Publishers, 1988.

[33] E. Roxin, "On finite stability in control systems," Rendiconti del Circolo Matematico di Palermo, vol. 15, pp. 273-283, 1966.

[34] H. K. Khalil, Nonlinear Systems, ser. NJ 07458. Upper Saddle River: Prentice-Hall, 1996.

[35] V. Zubov, "On systems of ordinary differential equations with generalized homogenous right-hand sides," Izvestia vuzov. Mathematica., vol. 1, pp. 80-88, 1958 (in Russian).

[36] L. Rosier, "Homogeneous Lyapunov function for homogeneous continuous vector field," Systems \& Control Letters, vol. 19, pp. 467-473, 1992.

[37] E. Bernuau, A. Polyakov, D. Efimov, and W. Perruquetti, "On extension of homogeneity notion for differential inclusions," in Proceeding of the European Control Conference, Zurich, 2013, pp. 2204-2209.

[38] G. Dahlquist and A. Björck, Numerical methods in scientific computing. SIAM, 2008.

[39] M. Livne and A. Levant, "Proper discretization of homogeneous differentiators," Automatica, vol. 50, no. 8, pp. 2007-2014, 2014. 